\title{
Deferoxamine Mesylate
}

National Cancer Institute

\section{Source}

National Cancer Institute. Deferoxamine Mesylate. NCI Thesaurus. Code C417.

The mesylate salt of an iron-chelating agent that binds free iron in a stable complex, preventing it from engaging in chemical reactions. Deferoxamine chelates iron from intra-lysosomal ferritin and ferrioxamine, a water-soluble complex excreted by the kidneys and in the feces via the bile. This agent does not readily chelate iron bound to transferrin, hemoglobin, myoglobin or cytochrome. 